\title{
Role of Multi-Detector Computed Tomography in Assessment of Blunt Abdominal Trauma in Pediatric Patients
}

\author{
AMINA I.E. ELKHOULY, M.Sc.; NAHED A. ABDULATIFE, M.D. and HEBA M. GALAL, M.D. \\ The Department of Radiodiagnosis, Faculty of Medicine (Girls), Al-Azhar University, Cairo, Egypt
}

\begin{abstract}
Background: Abdominal trauma in pediatrics is common. The delay in diagnosis and treatment could increase the complications rate. Using Computed tomography (CT) has been the gold standard in assessment of hemodynamically stable patients with blunt abdominal trauma.

Aim of Study: Was to identify the value of the MDCT in the assessment of different abdominal injuries as a result of blunt abdominal trauma in pediatricpatients.

Patient and Methods: This prospective study carried out on 50 pediatric patients with blunt abdominal trauma, those patients underwent multi-detector CT abdomen with intravenous contrast for detection of intra-abdominal injuries. This study was conducted between October 2018 and October 2019. The data collected were tabulated and analyzed statistically.

Results: MDCT study of pediatric patients was able to diagnose and grade solid and non-solid organ injuries. The spleen was the most commonly injured solid organ (56\%) followed by the liver (46\%) and kidney (32\%). MDCT findings were able to limit the need for surgical intervention, where most of the cases were managed conservatively.

Conclusions: MDCT candetect and accurately assess the grading of injuries of solid organs, non-solid hollow viscus organs. Also it perfectly diagnoses diaphragmatic injuries and bone fractures. Vascular and non-vascular complications were diagnosed accurately with triphasic contrast enhanced CT protocol. MDCT is of a great help in deciding the mostpreferable management by detecting life-threatening conditions such as the active bleeding. Also, it primarily suggests nonoperative management protocol as the intensity of care and the hospital stay duration.
\end{abstract}

Key Words: Multi-detector CT - Pediatric - Blunt traumaAbdomen.

\section{Introduction}

TRAUMA is a leading cause of morbidity and mortality in childhood, and blunt trauma accounts

Correspondence to: Dr. Amina I.E. Elkhouly, E-Mail: moony.elkholy@yahoo.com for $80-90 \%$ of abdominal injuries. The mechanism of trauma is quite similar to that of the adults, but there are important physiologic differences between children and adults in this field, such as the smaller blood vessels and the high vasoconstrictive response, leading to the spreading of a non-operative management [1].

The abdomen is the second most common site of injury. The most common reported mechanism for abdominal injury is motor vehicle crashes, followed by automobile-versus-pedestrian injuries and falls [2].

Pediatric abdominal trauma is common, with delays in diagnosis and treatment resulting in an increased rate of complications [3].

Advances in technology have made evaluation of intra-abdominal injuries increasingly less invasive, but clinical evaluation and an appropriate level of suspicion are the most important variables in management. Despite increased awareness and prevention efforts, trauma is the number one cause of death in the pediatric age group [3].

The role of the radiologist is to look for the following aspects that are to be expected in abdominal blunt trauma: Hemoperitoneum, contrast blush consistent with active bleeding, lacerations, contusions, parenchymal hematomas, subcapsular hematomas, devascularization of organs or parts of organs, free intraperitoneal or retroperitoneal air, diaphragmatic rupture [4].

Computed tomography (CT) is the gold standard in abdominal blunt trauma in hemodynamically stable patients [5], because it reduces the number of laparotomies. Also, ithelps to localize a hematoma and evaluate solid organ injuries [6]. 
The aim of the current study was to identify the value of the MDCT in the assessment of different abdominal injuries as a result of blunt abdominal trauma in pediatric patients.

\section{Patients and Methods}

This prospective study carried out on 50 pediatric patients with blunt abdominal trauma, those patients underwent multi-detector CT abdomen with intravenous contrast for detection of intraabdominal injuries.

Approval of the ethical committee of Al-Azhar Faculty of Medicine (Girls) and a written informed consent from all the subjects were obtained.

This study was conducted between October 2018 and October 2019.

Pediatric patients with blunt abdominal trauma were admitted to the Emergency Department at Damanhour Medical National Institute and referred to the Department of Radiodiagnosis.

All patients had undergone Multi-detector CT Abdomen and pelvis with intravenous contrast for detection of intra-abdominal injuries.

\section{Inclusion criteria:}

- Hemodynamically stable pediatric patients.

- Physical examination or laboratory findings suggestive of abdominal injury including hematuria, abdominal bruising or ecchymosis, abdominal distention, abdominal pain, absence of bowel sounds, vomiting, decreased hematocrit, and blood from the rectum or nasopharyngeal tube aspirate.

\section{Exclusion criteria:}

- Visible evidence of chest or abdominal injury.

- Age $<18$ years.

- Penetrating trauma.

- Blunt trauma not including the abdomen.

All the patients were subjected to the following:

1- Full history taking, thorough clinical examination.

2- Ultrasonography of the abdomen and pelvis.

3- MDCT for abdomen and pelvis: Using multidetector row CT (Toshiba 64). A pre contrast scan was done followed by IV contrast injection of non-ionic contrast material (Iopromide 300) with a dose of $1.5 \mathrm{mg} / \mathrm{kg}$.
- Scanning protocol for children was used by adjusting the peak kilovolt age and tube current settings (mAs) to minimize the radiation dose delivered to the pediatric patients. The examinations were done on 64-Toshiba MDCT scanner. The scanning parameters used were: $100 \mathrm{mAs}, 110 \mathrm{KVP}$, tube rotation time of $0.4 \mathrm{~s} /$ rot., $2 \mathrm{~mm}$ slice thickness and $1 \mathrm{~mm}$ reconstruction.

- Sedative intravenous thiopental sodium with a dose of $3 \mathrm{mg} / \mathrm{kg}$ was used in 5 patients in order to limit the excessive movements of these children and its effect on the multiplanar and reconstructive techniques.

4- The feedback of the medical and surgical data was obtained whenever available.

\section{Statistics:}

The data collected were tabulated and analyzed by SPSS (Statistical package for the social science software) statistical package version IBM compatible computer.

\section{Results}

This study involved 50 pediatric patients aged from 0-18 years old.

Age: Table (1) shows the age groups between the studied patients.

Table (1): The age groups of the studied patients.

\begin{tabular}{lllll}
\hline \multicolumn{5}{c}{ Age (years) } \\
\cline { 2 - 5 } & $0>5$ & $5>10$ & $10>15$ & $15>18$ \\
\hline Count & 4 & 16 & 18 & 12 \\
$\%$ & $8.0 \%$ & $32.0 \%$ & $36.0 \%$ & $24.0 \%$ \\
\hline
\end{tabular}

Sex: The study group consisted of 33 males and 17 females, Table (2).

Table (2): Sex variation among the studied patients.

\begin{tabular}{lll}
\hline & \multicolumn{2}{c}{ Sex } \\
\cline { 2 - 3 } & Male & Female \\
\hline Count & 33 & 17 \\
$\%$ & $66.0 \%$ & $34.0 \%$ \\
\hline
\end{tabular}

\section{Mode of trauma:}

Patients were subjected to various types of trauma. The commonest mode of trauma was motor vehicle accidents. Other modes of trauma are illustrated in Table (3). 
Table (3): Distribution of the studied cases according to mode of trauma.

\begin{tabular}{lll}
\hline Mode of trauma & Count & $\%$ \\
\hline Motor vehicle occupant & 25 & 50.0 \\
Pedestrian & 7 & 14.0 \\
Fall from a height & 13 & 26.0 \\
Sports injury & 1 & 2.0 \\
Child abuse & 3 & 6.0 \\
Cyclist & 1 & 2.0 \\
\hline
\end{tabular}

\section{Clinical presentations:}

Patients admitted to the emergency department presented by different clinical findings as shown in Table (4).

Table (4): Classification of pediatric patients accordingto the clinical presentations.

\begin{tabular}{lcl}
\hline Clinical presentations & Number of cases & $\%$ \\
\hline Abdominal pain & 39 & 78.0 \\
Hypotension & 34 & 68.0 \\
Nausea and vomiting & 33 & 66.0 \\
Abdominal ecchymosis & 16 & 32.0 \\
Hematuria & 11 & 22.0 \\
Jaundice & 4 & 8.0 \\
Fever & 8 & 16.0 \\
\hline
\end{tabular}

\section{Ultrasound findings:}

Ultrasound scan was performed to fifty stable pediatric patients. Findings are demonstrated in Table (5).

Table (5): Ultrasound findings in pediatric patients presented by blunt abdominal trauma.

\begin{tabular}{lll}
\hline Ultrasound findings & Number of cases $\%$ \\
\hline Free intra-peritoneal fluid collection & 44 & 88 \\
Retro-peritoneal fluid collection & 7 & 14.0 \\
Sentinel clot sign (Hemoperitoneum) & 4 & 8.0 \\
Splenic lacerations and contusions & 15 & 30.0 \\
Liver lacerations and contusions & 13 & 26.0 \\
Renal lacerations and contusions & 10 & 20.0 \\
Urinary bladder injury & 1 & 2.0 \\
Pancreatic injury & 1 & 2.0 \\
Supra-renal glands & 2 & 4.0 \\
Vascular complications & 3 & 6.0 \\
Pneumoperitoneum & 1 & 2.0 \\
$\quad$ (suspecting bowel injury) & & \\
\hline
\end{tabular}

\section{MDCT findings:}

Fifty pediatric patients admitted to the ER underwent Tri-phasic MDCT abdomen and pelvis; illustrated in Table (6).
Table (6): MDCT findings noted in paediatric patients admitted to the ER by blunt abdominal trauma.

\begin{tabular}{lll}
\hline MDCT findings & $\begin{array}{c}\text { Number of } \\
\text { pediatric patients }\end{array}$ & $\%$ \\
\hline - Free intra-peritoneal fluid collection & 41 & 82.0 \\
- Retro-peritoneal fluid collection & 9 & 18.0 \\
- Sentinel clot sign (Hemoperitoneum) & 15 & 30.0 \\
- Splenic lacerations and contusions & 28 & 56.0 \\
- Liver lacerations and contusions & 23 & 46.0 \\
- Renal lacerations and contusions & 16 & 32.0 \\
- Bowel injury (free fluid around & 3 & 6.0 \\
bowel loops, wall hematoma, & & \\
pneumoperitoneum) & & \\
- Urinary bladder injury & 2 & 4.0 \\
- Pancreatic injury & 4 & 8.0 \\
- Ureteric injury & 1 & 2.0 \\
- Supra-renal glands & 4 & 8.0 \\
- Diaphragmatic injury & 3 & 6.0 \\
- Vascular complications & 19 & 38.0 \\
$\quad$ (active contrast extravasation, & & \\
pseudoaneurysmal formation and & & \\
- arterio-venous fistula) & & \\
- Relvic Fractures & 5 & 10.0 \\
- Vertebral Fractures & 1 & 2.0 \\
- Vertes & 3 & 6.0 \\
\hline
\end{tabular}

\section{Splenic trauma:}

The CT grading of spleen injury and the Non Operative Management (NOM) failure in relation to CT grades illustrated in the following Fig. (1).

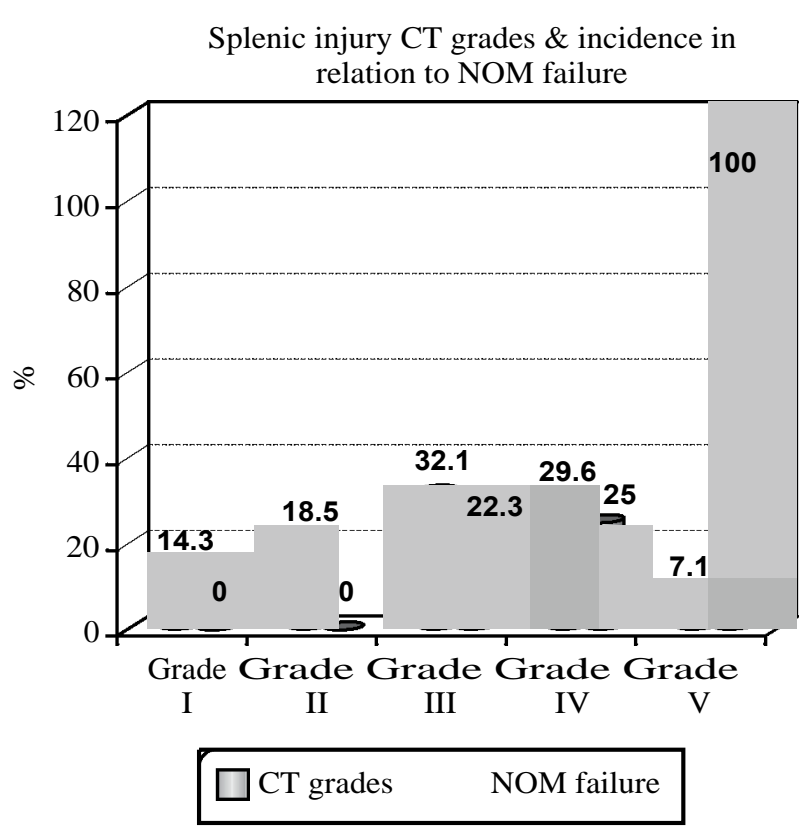

Fig. (1): Splenic injury CT grades \& incidence in relation to NOM failure $(n=28)$.

Complications of splenic injury in relation to CT grades of splenic trauma are shown in Table (7). 
Table (7): Incidence of associated splenic injury complications in relation to $\mathrm{CT}$ grades of splenic trauma.

\begin{tabular}{lcccccccc}
\hline & \multicolumn{8}{c}{ Complications } \\
\cline { 2 - 9 } $\begin{array}{l}\text { CT } \\
\text { grades }\end{array}$ & $\begin{array}{c}\text { Hemoperi- } \\
\text { toneum }\end{array}$ & $\begin{array}{c}\text { Pseudo- } \\
\text { aneurysm }\end{array}$ & $\begin{array}{c}\text { Arterio- } \\
\text { venous } \\
\text { fistula }\end{array}$ & $\begin{array}{c}\text { Active } \\
\text { extra- } \\
\text { vasation }\end{array}$ \\
\cline { 2 - 9 } & No. & $\%$ & No. & $\%$ & No. & $\%$ & No. & $\%$ \\
\hline Grade I & 0 & 0 & 0 & 0.0 & 0.0 & 0.0 & 0 & 0.0 \\
Grade II & 0 & 0 & 0 & 0.0 & 0 & 0.0 & 0 & 0.0 \\
Grade III & 0 & 0 & 0 & 0.0 & 0 & 0.0 & 1 & 11.11 \\
Grade IV & 4 & 50.0 & 2 & 25.0 & 0 & 0.0 & 5 & 62.5 \\
Grade V & 2 & 100.0 & 0 & 0.0 & 0 & 0.0 & 2 & 100.0 \\
\hline
\end{tabular}

\section{Liver trauma:}

The CT grading in hepatic injury and the Non Operative Management (NOM) failure in relation to CT grades illustrated in the following Table (8).

Table (8): Liver injury CT grades $\&$ incidence in relation to NOM failure $(n=23)$.

\begin{tabular}{lcccc}
\hline CT grades & Count & $\%$ & NOM failure & $\%$ \\
\hline Grade I & 3 & 13.04 & 0 & 0.0 \\
Grade II & 8 & 34.78 & 0 & 0.0 \\
Grade III & 8 & 34.78 & 1 & 12.5 \\
Grade IV & 3 & 13.04 & 2 & 66.67 \\
Grade V & 1 & 4.35 & 1 & 100.0 \\
\hline
\end{tabular}

Complications of liver injury in relation to CT grades of liver trauma are shown in Table (9).
Table (9): Incidence of associated liver injury complications in relation to $\mathrm{CT}$ grades of liver trauma.

\begin{tabular}{lcccccccc}
\hline & \multicolumn{8}{c}{ Complications } \\
\cline { 2 - 9 } $\begin{array}{l}\text { CT } \\
\text { grades }\end{array}$ & Bile leak & Biloma & $\begin{array}{c}\text { Re- } \\
\text { bleeding }\end{array}$ & $\begin{array}{c}\text { Liver } \\
\text { abscess }\end{array}$ \\
\cline { 2 - 10 } & No. & $\%$ & No. & $\%$ & No. & $\%$ & No. & $\%$ \\
\hline Grade I & 0 & 0.0 & 0 & 0.0 & 0 & 0.0 & 0 & 0.0 \\
Grade II & 0 & 0.0 & 0 & 0.0 & 0 & 0.0 & 0 & 0.0 \\
Grade III & 0 & 0.0 & 0 & 0.0 & 0 & 0.0 & 1 & 12.5 \\
Grade IV & 1 & 33.33 & 1 & 33.33 & 2 & 66.67 & 0 & 0.0 \\
Grade V & 1 & 100.0 & 0 & 0.0 & 1 & 100.0 & 0 & 0.0 \\
\hline
\end{tabular}

\section{Renal trauma:}

The CT grading in renal injury and the Non Operative Management (NOM) failure in relation to CT grades illustrated in the following Fig. (2).

Complications of liver injury in relation to CT grades of liver trauma are shown in Table (10).

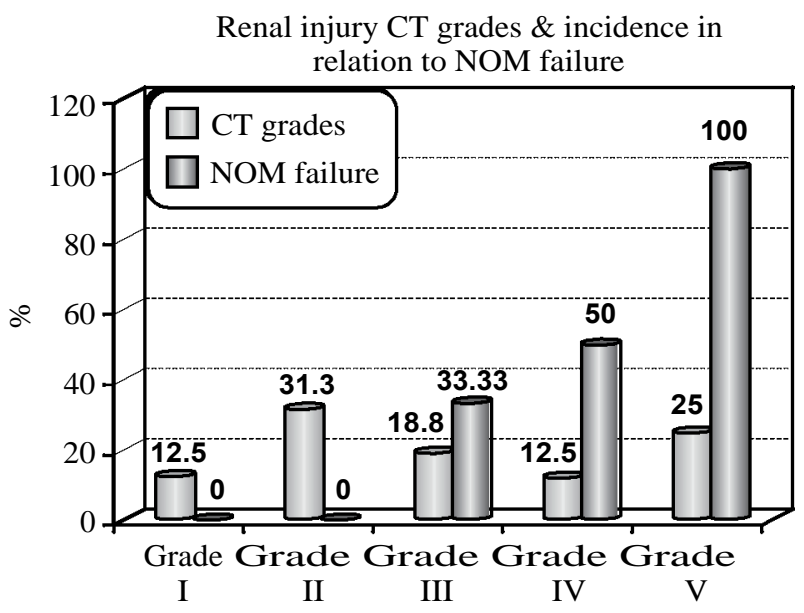

Fig. (2): Renal Injury CT grades \& incidence in relation to NOM failure $(n=16)$.

Table (10): Incidence of renal injury complications in relation to CT grades of Renal trauma.

\begin{tabular}{|c|c|c|c|c|c|c|c|c|c|c|c|c|}
\hline \multirow{3}{*}{$\begin{array}{l}\text { CT } \\
\text { grades }\end{array}$} & \multicolumn{12}{|c|}{ Complications } \\
\hline & \multicolumn{2}{|c|}{$\begin{array}{l}\text { Hemoperi- } \\
\text { toneum }\end{array}$} & \multicolumn{2}{|c|}{$\begin{array}{c}\text { Retro- } \\
\text { peritoneal } \\
\text { hematoma }\end{array}$} & \multicolumn{2}{|c|}{$\begin{array}{l}\text { Arterio- } \\
\text { venous } \\
\text { fistula }\end{array}$} & \multicolumn{2}{|c|}{$\begin{array}{l}\text { Pseudo- } \\
\text { aneurysm }\end{array}$} & \multicolumn{2}{|c|}{$\begin{array}{c}\text { Active } \\
\text { extravasation }\end{array}$} & \multicolumn{2}{|c|}{ Urinoma } \\
\hline & No. & $\%$ & No. & $\%$ & No. & $\%$ & No. & $\%$ & No. & $\%$ & No. & $\%$ \\
\hline Grade I & 0 & 0.0 & 0 & 0.0 & 0 & 0.0 & 0 & 0.0 & 0 & 0.0 & 0 & 0.0 \\
\hline Grade II & 0 & 0.0 & 0 & 0.0 & 0 & 0.0 & 0 & 0.0 & 0 & 0.0 & 0 & 0.0 \\
\hline Grade III & 1 & 33.3 & 1 & 33.3 & 0 & 0.0 & 0 & 0.0 & 0 & 0.0 & 0 & 0.0 \\
\hline Grade IV & 2 & 100.0 & 0 & 0.0 & 0 & 0.0 & 2 & 100.0 & 1 & 50.0 & 0 & 0.0 \\
\hline Grade V & 4 & 100.0 & 1 & 25.0 & 0 & 0.0 & 2 & 50.0 & 3 & 75.00 & 1 & 25.0 \\
\hline
\end{tabular}




\section{Bowel trauma:}

Bowel trauma injuries involving the jeujenum were detected in two cases, duodenal wall injury was detected in the third case. These injuries were suspected based on the presence of mild to marked free fluid collection between bowel loops, pneumoperitoneum and focal thickened bowel wall.

\section{Urinary bladder trauma:}

Urinary bladder injuries were detected in two patients. One patient had combined extraperitoneal and intraperitoneal rupture of urinary bladder. The other one showed intraperitoneal rupture of urinary bladder.

\section{Pancreatic trauma:}

Pancreatic injuries and late complications are illustrated in Tables $(11,12)$.

Table (11): Pancreatic Injury CT grades \& incidence in relation to NOM failure $(n=4)$.

\begin{tabular}{lcccl}
\hline CT grades & No. & $\%$ & NOM failure & $\%$ \\
\hline Grade I & 0 & 0.0 & 0 & 0 \\
Grade II & 2 & 50.0 & 1 & 50.0 \\
Grade III & 2 & 50.0 & 0 & 0 \\
\hline
\end{tabular}

Table (12): Incidence of pancreatic injury complications in relation to CT grades of pancreatic trauma.

\begin{tabular}{lclcl}
\hline & \multicolumn{4}{c}{ Complications } \\
\cline { 2 - 5 } CT grades & \multicolumn{2}{c}{ Pancreatitis } & \multicolumn{2}{c}{$\begin{array}{c}\text { Pancreatitic } \\
\text { pseudocyst }\end{array}$} \\
\cline { 2 - 5 } & No. & $\%$ & No. & $\%$ \\
\hline Grade I & 0 & 0.0 & 0 & 0 \\
Grade II & 1 & 50.0 & 0 & 0.0 \\
Grade III & 0 & 0.0 & 1 & 50.0 \\
\hline
\end{tabular}

Supra-renal glands injury:

Supra renal glands injury were noted in 4 patients; two showed right suprarenal gland injury and another two showed left suprarenal gland injury; in the four patients adrenal gland injury was demonstrated by non-enhancing hematoma replacing the supra-renal glands associated with diffuse enlargement of the adrenal glands and surrounding fat stranding.

\section{Ureter injury:}

One case with ureteric injury was associated with other organ injuries. MDCT findings of the ureteric injury were contrast spillage in delayed phase, with total amputation or attenuated filling of the ureter segments distal to the site of tear.

\section{Diaphragmatic trauma:}

MDCT demonstrated diaphragmatic tear in three patients. It was detected in the form of herniating intra-abdominal organs into the chest, with associated atelectasis of the ipsilateral lung and mediastinal shift. In two patients it showed ruptured right copula of the diaphragm with herniated liver into the chest, In the third patient it showed ruptured left copula of the diaphragm with herniated stomach into the chest and associated volvolus of the stomach.

\section{The type of successful management:}

MDCT findings were able to limit the need for surgical intervention, where most of the cases were managed conservatively, Table (13).

Table (13): Distribution of the studied cases according to the type of successful management.

\begin{tabular}{lcccc}
\hline \multirow{2}{*}{ Organ injuries } & \multicolumn{2}{c}{ Conservative } & \multicolumn{2}{c}{ Operative } \\
\cline { 2 - 6 } & Count & $\%$ & Count & $\%$ \\
\hline Spleen $(\mathrm{n}=28)$ & 16 & 57.14 & 12 & 44.44 \\
Liver $(\mathrm{n}=23)$ & 18 & 78.26 & 5 & 21.73 \\
Kidneys $(\mathrm{n}=16)$ & 9 & 56.25 & 7 & 43.75 \\
Supra renal $(\mathrm{n}=4)$ & 3 & 75.0 & 1 & 25.0 \\
Pancreatic injury $(\mathrm{n}=4)$ & 1 & 25.0 & 3 & 75.0 \\
Bowel injury $(\mathrm{n}=3)$ & 1 & 33.3 & 2 & 66.6 \\
Urinary bladder injury $(\mathrm{n}=2)$ & 0 & 0.0 & 2 & 100.0 \\
Ureteric injury $(\mathrm{n}=1)$ & 0 & 0 & 1 & 100.0 \\
Diaphragmatic tear $(\mathrm{n}=3)$ & 0 & 0.0 & 3 & 100 \\
Vascular complications $(\mathrm{n}=19)$ & 1 & 5.26 & 18 & 94.74 \\
Bone injuries $(\mathrm{n}=8)$ & 1 & 12.5 & 7 & 87.5 \\
\hline
\end{tabular}

\section{Illustrative Cases:}

A 12 years old male patient with blunt abdominal trauma by a motor vehicle accident. He was managed surgically.

A 10 years old female patient with blunt abdominal trauma by a fall from a height. She was managed operatively.

A 15 years old male patient with blunt abdominal trauma by falling from a height. He was managed conservatively. 

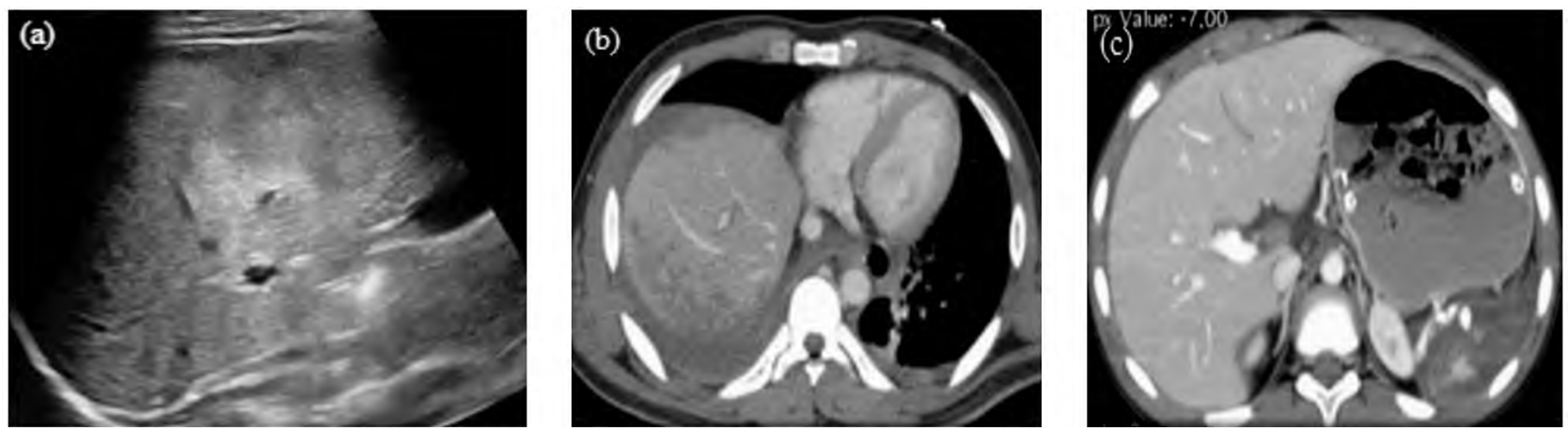

Fig. (3): (a) Abdominal Ultrasound scan revealed Free intra-peritoneal fluid collection. (b) Axial contrast enhanced CT scan portal phase CT demonstrates grade II liver laceration. Right pleural collection. (c) Portal phase reveal Grade V splenic injury associated with hyper dense foci of contrast extravasation at the splenic hilum which matches the active bleeding.
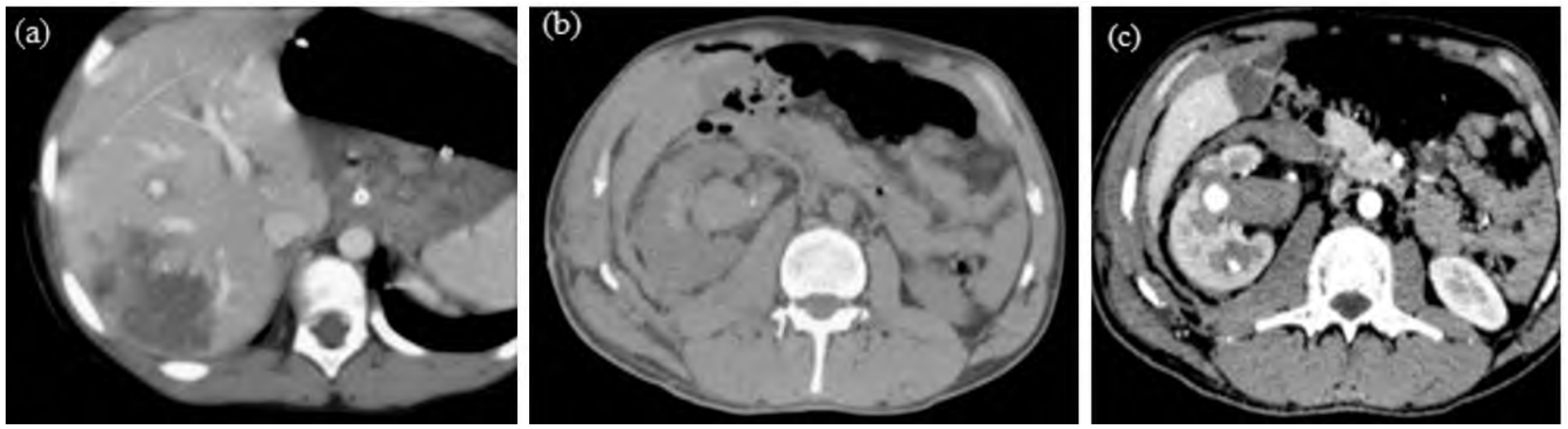

Fig. (4): (a) Axial contrast enhanced CT scan of the abdomen, Portal phase revealed non-enhancing hepatic area denoting grade III liver injury. (b) Plain CT axial abdomen revealed right kidney intra-calyceal hematoma. (c) Axial contrast enhanced CT scan of the abdomen, arterial phase demonstrated linear non enhancing right renal laceration seen reaching the pelvis associated with contained blood formation representing grade $\mathrm{V}$ right renal injury with pseudo-aneurysmal formation.
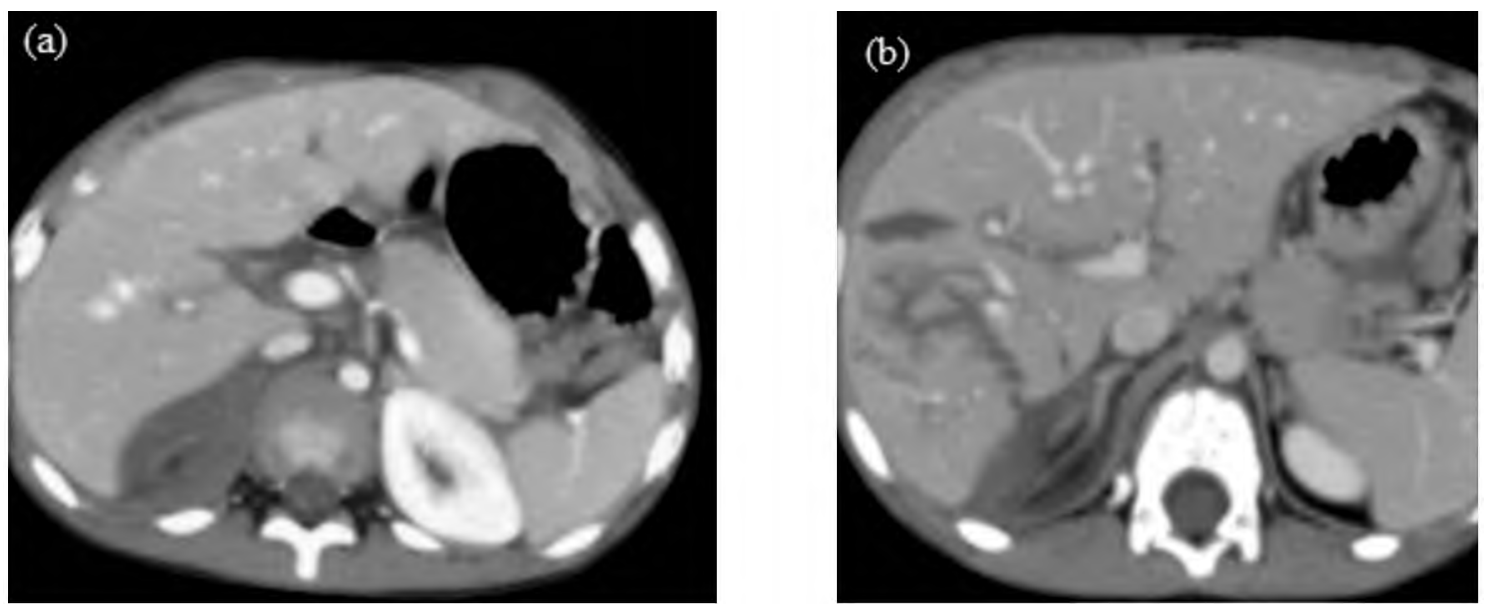

Fig. (5): (a) Contrast enhanced axial CT scan of the abdomen, arterial phase demonstrates enlarged right adrenal gland with minimal detected enhancement associated with hematoma replacing most of the gland. (b) Contrast enhanced axial CT scan of the abdomen, venous phase show grade III liver laceration and right suprarenal gland injury.

\section{Discussion}

Injury and violence is a major killer of children throughout the world, responsible for about 950 000 deaths annually, in children and young people under the age of 18 years. The World Health Organization reported the majority of the deaths
$(90 \%)$ were due to unintentional injuries such as road traffic injuries (RTI), drowning, burns and poisoning [7].

Childhood injury is a major public health problem that requires urgent attention. The problem is more common in low and middle income countries 
(LMIC) than in high income countries (HIC) with variations according to type of injury [8].

This study showed that the prevalence of blunt abdominal trauma is higher in males $(66 \%)$ than in females which agrees with Bulus $\mathrm{J}$ et al. [9] who state that Boys $(62.1 \%)$ are at an increased risk of injury than girls, possibly because boys are more adventurous than girls.

The present study showed that children from five to fifteen years old are more vulnerable to blunt abdominal trauma. This agrees with Hyder AA et al. [10] in the GCUIS who state that (60\%) of the total cases were $\geq 5$ years of age.

This is in accordance with Muriu $\mathrm{N}$ et al. [7] who state that prevalence of injury was noted to increase with advancing age $(52.3 \%)$ in children were $\geq 5$ years of age.

In our study it was noted that RTAs (road traffic accidents), including motor vehicle collision accidents and pedestrian represent the commonest mode of trauma (64\%), followed by falls from height which represents $24 \%$.

This agreed with Bulus $\mathbf{J}$ et al. [9] who state that the commonest childhood injury is RTI which accounted for $56.9 \%$ followed by fall $23.6 \%$.

Basaran A et al. [11] state that among injury mechanisms, motor vehicle accidents were the most common with a percent of $41.4 \%$, and falling $19.7 \%$ was the second most common.

In our study the clinical findings on admission were mainly abdominal pain followed by hypotension, nausea and vomiting. Some patients were presented by more than one clinical picture. This matches the study of Basaran A et al. [11] who clarified that the most common physical examination finding in patients was tenderness at $67 \%$.

Ultrasound was more sensitive in the detection of abdominal fluid collection compared with the MDCT findings. In our study ultrasound detected intra-peritoneal fluid collection in 44 patients, also retro-peritoneal fluid collection in 7 patients and hemoperitoneum in 4 patients.

As compared to the MDCT findings, ultrasound showed limited sensitivity in the evaluation of solid organ injury and hollow viscus organs such as the colon, small bowel and ureters. It was not able to evaluate bone fractures and Diaphragm injuries.

This matches the study done by Nnamonu M.I et al. [12] who declaredthat ultrasound has a high positive predictive value in screening for free intraperitoneal fluid and when scanning for the visceral parenchymal injury, the Positive Predictive Value was low.

This also agreed with Botros SM et al. [13] they declared that hepatic lacerations or hematomas, pancreatic and gastrointestinal injuries are difficult to see by ultrasonography.

Our study shows that MDCT was able to accurately diagnose and evaluate the grading of solid organ injuries as well as hollow viscus organ injuries, ureteric injuries, diaphragmatic tear and bone fractures. Triphasic contrast enhanced CT was able to diagnose vascular complications and nonvascular complications (abscess formation, biliary complications and pseudo pancreatic cystic formation).

Botros SM et al. [13] also state that Patients with solid organ injuries or hollow viscus organ injuries who are hemodynamically stable could perform CT abdomen for accurate characterization of their injuries and evaluation of different injury grades. They declared that Contrast enhanced computed tomography (CT) is the radiological golden standard for abdominal visceral injuries.

This matches the study of Basaran A et al. [11] who clarified that $\mathrm{CT}$ is an important imaging method for detecting solid organ injuries, classifying the injuries and determining the indicated treatment type.

In the present study, The spleen was the most commonly injured solid organ in patients. Triphasic $\mathrm{CT}$ was able to diagnose splenic injuries in $56 \%$ of pediatric patients, their different grades according to American Association for Surgery of Trauma (AAST) grading scales and the related complications, it was found that patients with higher grades of splenic injury developed more complications than others with low grade splenic injury. CT allowed conservative management as a first line of treatment in Grade I, II and III splenic injury, The NOM shows failure in two patients $(22.22 \%)$ with grade III splenic injury, one of them had active contrast extravasation. The NOM failure was noted in two patients $(25 \%)$ with Grade IV, one of them had hilar pseudo aneurysm and minimal enhancing spleen tissue, the other had a huge hematoma compressing the residual minimally enhancing spleen tissue and active contrast extravasation. Also, NOM failure was noted in two patients (100\%) presented by Grade V showing nonenhancing splenic tissue complicated by hemoperitoneum and active extravasation. Sixteen patients 
with splenic injury were managed conservatively $(57.1 \%)$, while operative management was done in twelve patients representing (44.4\%).

Agreeingwith Wisner et al. [14] who investigated a total 605 children with solid organ injuries after blunt torso trauma and found spleen injury as the most common injuried organ in $49 \%$ of these children. And disagreeing with Basaran A et al. [11] showed that the most commonly injured organ $(n=320)$ was the liver in $47 \%$ of patients followed by injury of the spleen in $36 \%$.

In the present study, The liver was the second injured solid organ in $46 \%$ of patients. Tri-phasic $\mathrm{CT}$ was able to diagnosehepatic injury as well as their different grades according to American Association for Surgery of Trauma (AAST) grading scales and the related vascular or non vascular complications, it was noted that patients with higher grades of liver injury developed more complications than others with low grade liver injury. This grading and its associated complications could not be identified using any other modality which allowed to predict the success of the NOM protocol.

Three patients showed grade I liver injury (13.04\%), eight patients showed grade II liver injury (34.78\%). Tri-phasic CT showed no biliary or vascular complications in those grades that allowed total recovery after NOM protocol. Eight of patients presented by grade III liver injury $(34.78 \%)$, seven were treated conservatively, one patient had late liver sub capsular abscess formation. Three patients had Grade IV liver injury $(13.04 \%)$, one treated conservatively, the other two showed vascular and biliary complications which was successfully managed operatively. One patient had Grade V liver injury $(4.35 \%)$ and was managed surgically due to uncontrolled bleeding. In total of 23 patients with liver injuries operative management was undertaken in only 5 patients representing only $21.73 \%$.

This agreeing with Wisner et al. [14] who state that the liver was the second commonly injured organ in $47 \%$ of patients.

And with Fodor M et al. [15] who found thatMost liver injuries are grade I, II or III, and are successfully treated conservatively. In contrast, the majority of grade IV or V liver injuries necessitate operative intervention. The success rate of NOM of hepatic trauma ranges from 82 to $100 \%$.

The kidney was the third most common injured organ after blunt abdominal trauma. Tri-phasic CT played important role in assessment of renal injuries, and its associated complications. sixteen patients $(32 \%)$ presented by renal injuries.

Two patients (12.5\%) presented by Grade I renal injury, another five patients $(31.25 \%)$ presented by grade II and was successfully recieved conservative management. Three patients $(18.75 \%)$ presented by grade III renal injury, two of them were managed conservatively, and one patient presented by hemoperitoneum and was managed operatively. Two patients $(12.5 \%)$ had grade IV renal injury, complicated by hemoperitoneum, pseudo aneurysm formation, and active bleeding with large perinephric hematoma and was managed surgically. Four patients $(25 \%)$ with grade $\mathrm{V}$ renal injury, one showed main renal artery complete amputation, another one showed renal laceration involving the pelvic-ureteric junction and the other two showed hemoperitoneum and pseudoaneurysmal formation, they were managed operatively. From sixteen patients presented by renal injuries, only seven patients $(43.75 \%)$ were managed operatively.

Wisner et al. [14] also state that the kidney was the third commonly injured organ in $32 \%$ of patients.

In this study, two patients were diagnosed as jeujenal injury representing the most common site of bowel injury and were managed surgically. One patient presented with third part duodenal wall injury with intraluminal and retroperitoneal hematoma that was managed conservatively.

In our study it was found that the most accurate CT finding for the diagnosis of bowel injury is mild to marked amount of hemoperitoneum noted between bowel loops provided that there is no other source of active bleeding. Two patients $(66.67 \%)$ in the present study showed mild and moderate amount of free collection between bowel loops denoting small bowel injury. Other CT findings that may suspect bowel injury are the presence of pneuo-peritoneum which was detected in two patients $(66.67 \%)$. Three patients $(100 \%)$ in our study showed focal hyper-dense wall thickening, representing focal intramural hematoma.

Also with Parambath A et al. [16] in the study of twenty-nine patients who were identified with bowel and mesenteric injury and underwent MDCT. Multi-detector CT is an excellent diagnostic modality in bowel and mesenteric injury. Thinner section imaging, fast acquisition of data, multiplanar reformation capability and increased awareness about various CT findings are the main con- 
tributory factors for better diagnostic efficacy of MDCT. Percentages of cases showing different findings are: Intraperitoneal fluid (89\%), bowel wall thickening (48\%), extra luminal air (24\%), retroperitoneal fluid (13.7\%).

CT was able to differentiate between three types of urinary bladder injury by the spillage of contrast either intra peritoneal, extra peritoneal or combined. This was important in choosing the appropriate management. One patient, who had combined urinary bladder injury was managed by surgical management. The other one who showed intraperitoneal urinary bladder injury was managed surgically.

This agreed with the study of Miele V et al. [1] where they declared that MDCT remains still the technique of choice in urinary tract injuries, because of its high sensitivity and accuracy, helping to discriminate between an intra-peritoneal form a retroperitoneal urinary leakage, requiring two different managements.

Also with Pereira BM et al. [17] who found that Surgical repair was the treatment in 101 cases $(90.9 \%)$ with intraperitoneal bladder rupture in their study.

In the present study; the $\mathrm{CT}$ grades of pancreatic injury, takes in consideration mainly the involvement of the pancreatic duct which is the main consideration in directing management. Two patients with grade II injury, one of them was managed conservatively and the other one was managed by distal pancreatectomy due to associated spleen Grade V trauma. Two patients showed grade III pancreatic injury, one of them was missed at first and was readmitted by complicated missed pancreatic injury in the form of pancreatic pseudocyst which was managed operatively and the other patient had suspected pancreatic ductal injury that was managed operatively.

This coincides with Antonsen I et al. [18] who state that pancreatic trauma resulting from blunt abdominal injuries in children are less common than injuries to the spleen, liver and kidneys. However, children with pancreatic injuries often have injuries to other organs. Computed tomography is the first choice in diagnostics, and is more widely used and has better sensitivity over ultrasound. Less severe (grade I-II) pancreatic injuries are treated conservatively. The choice of surgery or conservative treatment of severe injuries (grade III-V) where the pancreatic duct is involved must be considered for each individual patient.
Ureteric injury was noted in one patient associated with grade $\mathrm{V}$ renal injury and diagnosed by tri-phasic CT in the excretory phase which showed active spillage of contrast from the torn ureter that was diagnostic for ureteral injury and was managed operatively.

Agreeing with Iwase et al. [19] who state that the excretory phase of a contrast-enhanced CT scan of the kidney, ureters and bladder is recommended in diagnosis of ureteric injuries and a high index of suspicion must be maintained because delayed diagnosis results in higher complication rates. Ureteral injures are frequently missed due to haemodynamic instability and other associated injuries. It is important that trauma specialists recognize additional injuries after major trauma to prevent complications.

In the current study, Supra renal injury was not an isolated organ injury, it was associated with other organ injuries. Three patients presented with supra-renal injuries were managed conservatively and one patient was managed operatively.

This agreed with the study of Miele V et al. [1] where they declared that CT is the gold standard exam in detecting traumatic adrenal gland lesions. Main CT details are hematoma around 60-83\%, overall adrenal hemorrhage around $9-43 \%$, the homogeneous swelling of the adrenal gland around $10 \%$, and the adrenal rupture that is very rare.

Also agreed with Liao $\mathrm{CH}$ et al. [20] who explained that adrenal gland trauma is treated by conservative measures.

In our study, MDCT was able to diagnose diaphragmatic tear in three children and they were managed operatively.

Okur $\mathrm{M}$ et al. [21] also state that MDCT including multiplanar reconstruction or volume rendering, can be helpful to verify the diagnosis of diaphragmatic injuries and detect associated injuries. Operative strategies should be planned based on the localization, size of the defect, associated injuries and stability of the patient.

\section{Conclusion:}

MDCT was able to accurately diagnose and evaluate the grading of solid organ injuries, hollow viscus organ injuries, ureteric injuries, diaphragmatic tear and bone fractures. By using triphasic contrast enhanced protocol, vascular complications were diagnosed accurately, as well as non-vascular complications. 
MDCT can help prioritize optimal management by diagnosing life-threatening injury and active hemorrhage. MDCT primarily guides nonoperative decision making, such as intensity of care and duration of hospitalization.

Although the decision to operate is usually depend on clinical findings rather than radiological findings, MDCT evaluation increases diagnostic confidence and reduces rates of unnecessary laparotomies.

MDCT imaging remains the gold standard for the diagnosis of IAI, but should be used judiciously due to the risk of radiation-induced malignancy.

\section{References}

1- MIELE V., PICCOLO C.L., TRINCI M., GALLUZZO M., IANNIELLO S. and BRUNESE L.: Diagnostic imaging of blunt abdominal trauma in pediatric patients. Radiol Med. May, 121 (5): 409-30, 2016. doi: 10.1007/s11547016-0637-2.

2- MILROY C.M.: Blunt Abdominal and Thoracic Injuries in Children. In: Collins K., Byard R. (eds) Forensic Pathology of Infancy and Childhood. Springer, New York, NY, 2014.

3- TERRENCE CURRAN, YOUNG HWAN CHUN, ANN DIETRICH, TARYN TAYLOR and LEE ANN WURSTER: Blunt Abdominal Trauma in Pediatrics: Pediatric Trauma Care II: A Clinical Reference for Physicians and Nurses Caring for the Acutely Injured Child. AHC Media LLC. Atlanta, GA, Chapter 4: 27-36, 2014.

4- ADEL MOHAMED EL WAKEEL, REHAB MOHAMED HABIB and ABEER NABIL ALI: Role of CT in Evaluation of Blunt Abdominal Trauma. International Journal of Medical Imaging, Vol. 3, No. 5, pp. 89-93, 2015. doi: 10.11648 /j . ij mi .2015 03 05.11.

5- WORTMAN J.R., UYEDA J.W., FULWADHVA U.P. and SODICKSON A.D.: Dual-Energy CT for Abdominal and Pelvic Trauma. Radiographics. Mar-Apr., 38 (2): 586$602,2018$.

6- DAYAL M., GAMANAGATTI S. and KUMAR A.: Imaging in renal trauma. World J. Radiol., 5 (8): 275-84, 2013.

7- MURIU N., KARANJA P. and KARAMA P.: Prevalence and factors associated with injuries among children aged 12 years and below admitted to hospitals in Nyeri County, Kenya. Journal of Health, Medicine and Nursing, 1 (1): 33-51, 2017. Retrieved from https://www.iprjb.org/journals /index.php/JHMN/article/view/272.

8- SLEET D.A.: The Global Challenge of Child Injury Prevention. International Journal of Environmental Research and Public Health, 15 (9): 1921, 2018.

9- BULUS J., LAR N., REMON L., INYANG B., SHU'AIBU J. and ABOIYAR A.: Predictors of unintentional childhood injuries seen at the Accident and Emergency Units of three tertiary health care centres in Jos. Int. J. Med. Biomed Res., 4 (3): 127-134, 2015.

10- HYDER A.A., SUGERMAN D.E., PUVANACHANDRA P., et al.: Global childhood unintentional injury surveillance in four cities in developing countries: A pilot study. Bull World Health Organ, 87 (5): 345-352, 2009. doi:10.2471/ blt.08.055798.

11- BASARAN A. and OZKAN S.: Evaluation of intraabdominal solid organ injuries in children. Acta. Biomed, 89 (4): 505-512, 2019. Published 2019 Jan 15. doi: 10.23750/abm.v89i4.5983.

12- NNAMONU M.I., IHEZUE C.H., SULE A.Z., RAMYIL V.M. and PAM S.D.: Diagnostic value of abdominal ultrasonography in patients with blunt abdominal trauma. Niger. J. Surg. Res., 19: 73-78, 2013.

13- BOTROS MALAK, SAMER, NASSEF, MOHAMED, ABDEL-GHANY and AHMED: Blunt abdominal trauma: The role of focused abdominal sonography in assessment of organ injury and reducing the need for CT. Alexandria Journal of Medicine, 12. 10.1016/j.ajme.2015.02.001, 2015.

14- WISNER DAVID, KUPPERMANN NATHAN, COOPER ARTHUR, MENAKER JAY, EHRLICH PETER, KOOISTRA JOSH, MAHAJAN PRASHANT, LEE LOIS, MSTAT LAWRENCE, YEN KENNETH, LILLIS KATHY and HOLMES JAMES: Management of children with solid organ injuries after blunt torso trauma. The journal of trauma and acute care surgery, 79: 206-214, 2015. 10.1097/TA.0000000000000731.

15- FODOR MARGOT, PRIMAVESI FLORIAN, MORELLHOFERT DAGMAR, HASELBACHER MATTHIAS, BRAUNWARTH EVA, CARDINI BENNO, GASSNER EVA, ÖFNER DIETMAR and STÄTTNER STEFAN: Non-operative management of blunt hepatic and splenic injuries-practical aspects and value of radiological scoring systems. European Surgery, 10.1007/s10353-018-0545$\mathrm{x}, 2018$.

16- PARAMBATH ARIF, HILILI SHATHA and RAVIKUMAR VAJJALLA: Multi-detector CT (MDCT) in bowel and mesenteric injury. Journal of Emergency Medicine, Trauma and Acute Care, 2. 10.5339/jemtac.2013.2, 2013.

17- PEREIRA B.M., OGILVIE M.P., GOMEZ-RODRIGUEZ J.C., et al.: A review of ureteral injuries after external trauma. Scand J. Trauma Resusc Emerg. Med., 18: 6, 2010. Published 2010 Feb 3. doi:10.1186/1757-7241-186.

18- ANTONSEN INGRID, BERLE VERONICA and SØREIDE KJETIL: Blunt pancreatic injury in children. Tidsskrift for den Norske laegeforening: Tidsskrift for praktisk medicin, ny raekke, 137. 10.4045/tidsskr.16.0888, 2017.

19- IWASE FUMIAKI, MIYAZAKI YOSHIBUMI, KOBAYASHI TASTUHO, KIKUCHI HIROKO and MASTUDA KIYOSHI: Bilateral ureteropelvic disruption following blunt abdominal trauma: Case report. BMC Urology, 11. 14. 10.1186/1471-2490-11-14, 2011.

20- LIAO C.H., OUYANG C.H., FU C.Y. and WANG C.Y.: The current status and management of blunt adrenal gland trauma. World J. Sur., 157: 338-43, 2015.

21- OKUR MEHMET, UYGUN IBRAHIM, ARSLAN MEHMET, AYDOGDU BAHATTIN, TÜRKOGLU AHMET, GOYA CEMIL, ICEN MUSTAFA, CIGDEM MURAT, ONEN ABDURRAHMAN and OTCU SELCUK: Traumatic diaphragmatic rupture in children. Journal of pediatric surgery, 49: 420-3, 2014. 10.1016/j.jpedsurg.2013. 11.056 . 


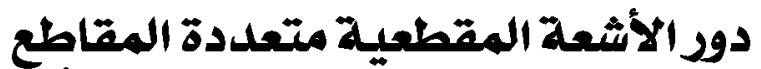

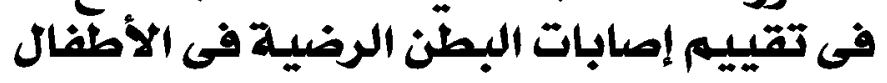

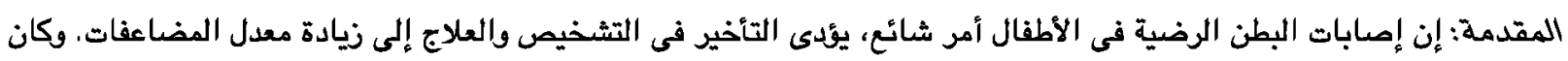

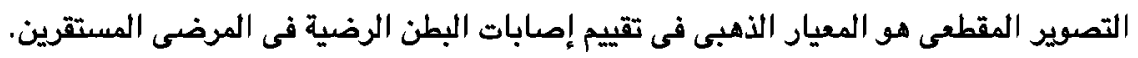

الفرض من البحث: هو دراسة قيمة الأشعة المقطعية متعددة المقاطع فى تقييم إصابات البطن المختلفة والناتجة عن صدمة البطن الرضية فى مرضى الأطفال.

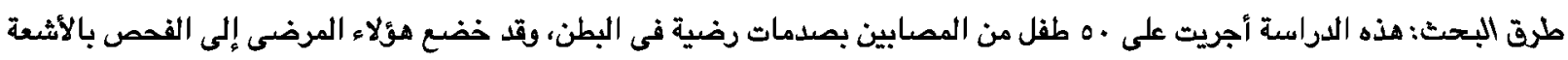

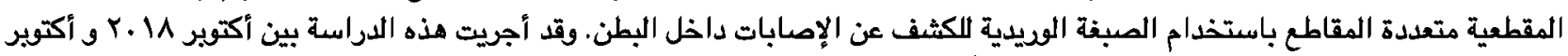

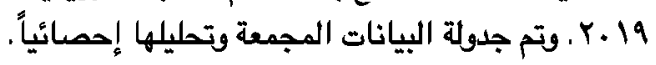

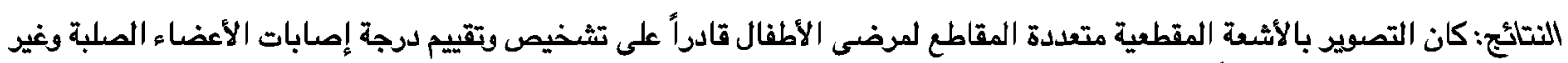

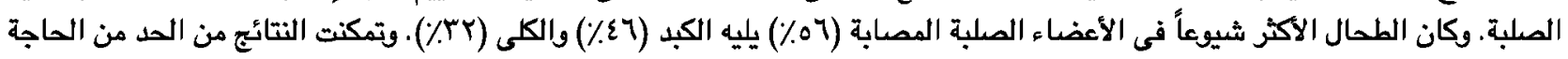
إلى التذخل الجراحى، حيث تم علاج معظم الحالات بدون جراحة.

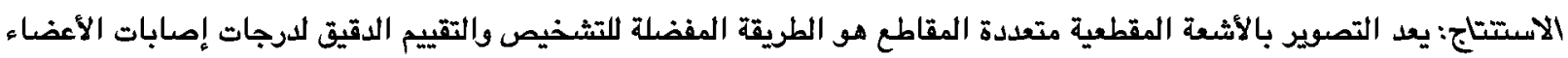

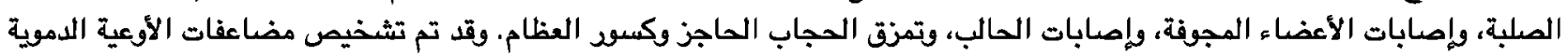

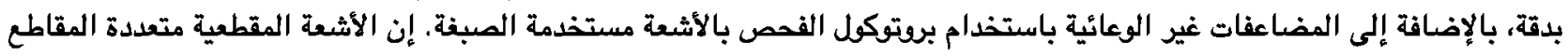

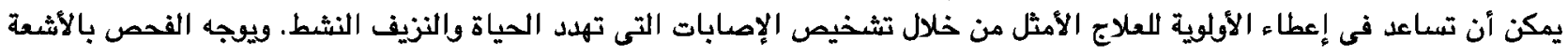

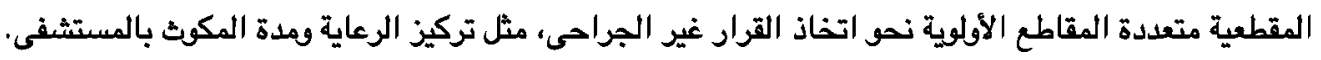

